\title{
Simulation-based particle swarm optimization technique for the breadth design of stairs and passageway network at interchange stations
}

\author{
Afaq khattak* \\ Department of Civil Engineering, Faculty of Engineering and Technology, International Islamic \\ University, Islamabad, Pakistan
}

\begin{abstract}
This study reports a novel hybrid simulation based Particle Swarm Optimization (PSO) technique using PH-Distribution for the design of facilities at transit stations. First, a Discrete-Event Simulation (DES) model for the network of stairs and passageway is developed that incorporates all influential factors that were ignored by the existing design codes (TCRP Report-165). The DES model is coupled with the PSO technique to build an automated framework for the area of network of stairs and passageway. The experimental results by hybrid simulationoptimization reveal that: (1) increase in randomness (SCV) of passengers' arrival causes increase in the breadth of both passageways and stairs; 2) For the same arrival rate and SCV, the stairs breadths are more than passageway breadths; 3) The breadth decreases from Level of Service (LOS) B towards D.
\end{abstract}

\section{Introduction}

The Transit Capacity and Quality of Service Manual by Transit Cooperative Research Program (TCRP Report-165) [1] provide the techniques for the design of service facilities at transit stations. However, the techniques neglect several influential factors as a result of which the congestion issues occur during peak and off-peak hours. Hu et al., [2] developed a stochastic analytical model to prevail over the deficiencies in the TCRP Report-165 devised a technique to design the breadth of the corridor facility, but it is applicable to a single facility and cannot be applied to the network of facilities. Our paper reports in this regard a coupled simulation- Particle Swarm Optimization (PSO) technique using PhaseType (PH) Distribution for the breadth design of network of stairs and passageways. This proposed model considers the fluctuation in the arrival flow of passengers at the transit area when passengers transfer from the platform of one line to another in the interchange station. Moreover, the blocking phenomenon is also incorporated in the proposed simulation-PSO technique that keeps the probability below a certain tolerance value.

Several studies describe the pedestrian and vehicular service facilities as the queuing systems. For instance, the G/M/1 analytical queuing model [3] an $\mathrm{M} / \mathrm{G}(n) / \mathrm{N} / \mathrm{N}$ network simulation model [4-5] and subway station capacity using $\mathrm{M} / \mathrm{G}(n) / \mathrm{N} / \mathrm{N}$ [6]. The major

\footnotetext{
*Corresponding author: afaq.khattak@iiu.edu.pk
} 
downside of these existing models is the implication of exponential distribution, which is only apt for a free flow conditions. The Squared Coefficient of Variation (SCV) of exponential distribution is 1 , which shows that fluctuation in passengers' arrival and service processes is disregarded, but practically the randomness exists. Recently, several domains such as computer communication systems [7], modeling health care system [8], etc have replaced exponential distribution with the Phase-Type (PH) distribution.

\section{Network Representation of stairs and passegeway}

The stairs and passageway the transit station form an open queuing network system. The passengers' enter the circulation area (stairs) as they alight the train both at Line 1(route 1) and Line 2 and exit at the platform of Line 3, as shown in figure 1. Passengers traverse the circulation area in certain amount of time which is knows as dwell time. The passengers' circulation in figure 1 can be represented as a merging topology of a queuing network system (figure 2). The stairs and passageway are the nodes of a queuing network, which is represented by $j=1,2, \ldots Z$. Each stair and passageway has finite capacity and passengers are blocked when arrival demand exceed the capacity. For simplicity, a uni-direction flow passenger at interchange station is considered. The service time of stairs and passageways is relation to the number of passengers in it. The walking speed of passengers drops as the number of passenger in the circulation area increases, which is known as the statedependence. Based on this analysis, each passenger's circulation area (Stairs and Passageways) network is represented by $X^{j} / Y(n)^{j} / Z^{j} / Z^{j}$ queuing system. Here $X^{j}, Y(n)^{j}$ and $Z^{j}$ are the random variable for passengers' arrival, circulation area state-dependent service time and capacity of any $j^{\text {th }}$ passengers' circulation area, respectively.

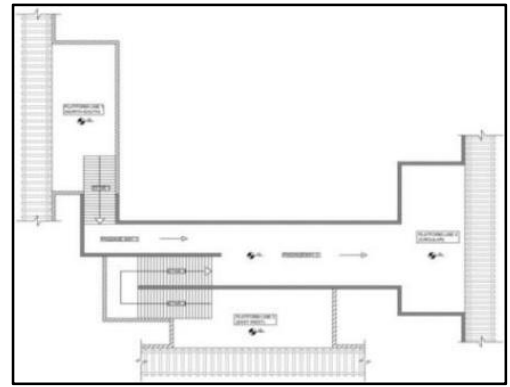

Fig. 1. Layout of a three-level interchange station.

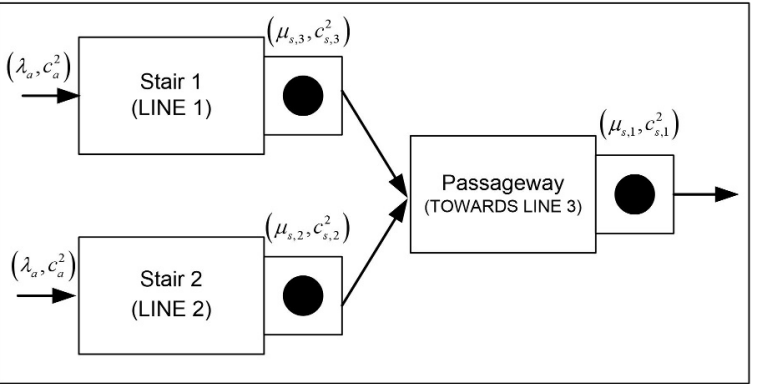

Fig. 2. Merging topology representation of stairs and passageway

\subsection{Random Variates from PH Distribution}

The proposed DES model involves generation of $\mathrm{PH}$ random variates from the $\mathrm{PH}$ distributions by using 2-tuple (Phase-Type representatives). We have used Neuts' Count Technique [9] for the generation of $\mathrm{PH}$ random variates.

\section{Automated simulation-PSO technique for the breadth design of network}

As discussed earlier, in this research we develop a DES model based on the PHDistribution in SimEvents ${ }^{\circledR}$ module. The PH-based DES model of network of stairs and passageways is integrated with $M A T L A B$ programming environment that contains the optimization script of PSO. Both DES model and MATLAB optimization script in 
conjunction provide the optimal breadths of stairs and passageway. The two major stages in proposed DES model are passengers' arrival stage; state-dependent service stage as shown in figure 3.

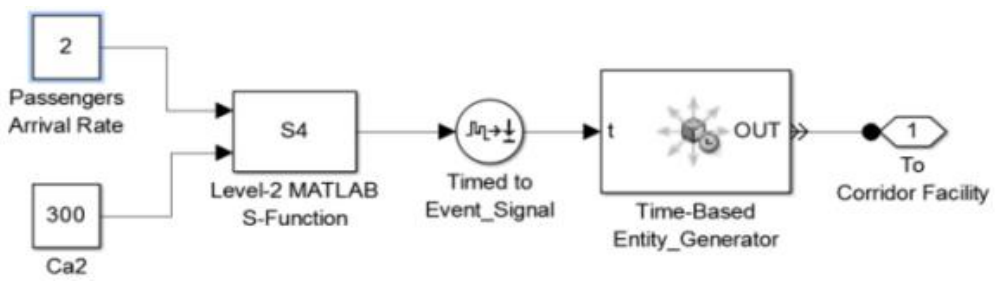

(a) Passengers' arrival stage

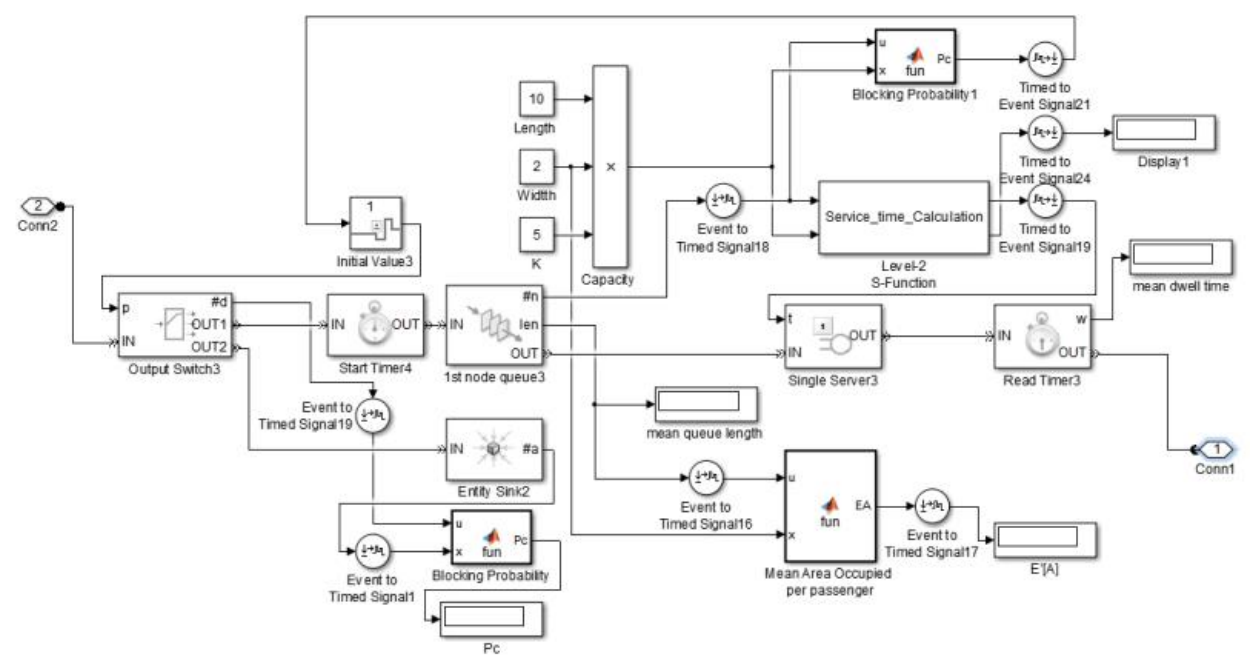

(b) State-dependent service stage

Fig. 3. SimEvents ${ }^{\circledR}$ architecture of passengers' circulation area at interchange station.

\subsection{PH-based Passengers Arrival Stage}

The Neuts' Count Technique for the generation of $\mathrm{PH}$ random variates takes the passengers' arrival rate arrival rate $\lambda_{a}$ and $S C V$ as two inputs (Phase-Type representatives). During the design stage of interchange station, $\lambda_{a}$ and $S C V$ are obtained from the peak-hour volume $(\mathrm{Q})$, peak-hour factor $(\varepsilon)$ and the average headway $\left(h_{a}\right)$ between the successive trains. The $\lambda_{a}$ is obtained by using the equation (1)

$$
\lambda_{a}=\frac{Q}{3600 \varepsilon}
$$

Similarly, SCV can be obtained by using equation (2).

$$
S C V=\frac{Q h}{3600 \varepsilon}-1
$$

\subsection{PH-based State-dependent Service Stage}

The passengers' walking speed is associated with the mean dwell time $\left(T_{d}\right)$ of passengers' in the network of stairs and passageway. The $T_{d}$ increases with decrease in walking speed. 
The state-dependent service time can be related to the state-dependent flow rate by using following equation (3).

$$
T_{s}(n)=1 / \mu_{s}(n)=L / n S(n)
$$

Similarly, state-dependent service rate is given by equation (4);

$$
\mu_{s}(n)=n S_{1} \exp \left[-\left(\frac{n-1}{v}\right)\right]^{\gamma} / L(i=1,2 \ldots Z)
$$

To employ this state-dependence condition in SimEvents ${ }^{\circledR}$ module (shown in figure 3), the passengers generated at the arrival stage stay in the FIFO Queue block prior to being served by Single Server block. The MATLAB ${ }^{\circledR}$ Function blocks in the sub-model are used to conduct several tasks: The block computes the mean area per passenger (E[A]) on stairs and passageway. It also compares the number of arrival with the stairs and passageway capacities and avert the passengers to enter when capacity is attained i.e., when $n=N$. The blocking probability $\left(P_{c}\right)$ of passengers in the stairs and passageways is also calculated by this block.

\subsection{Hybridization of PSO with DES model}

The proposed framework of DES model coupled with Particle Swarm Optimization (PSO) [13] is developed for the computation of optimal breadths of circulation areas. The integrated framework is described by figure 4 .

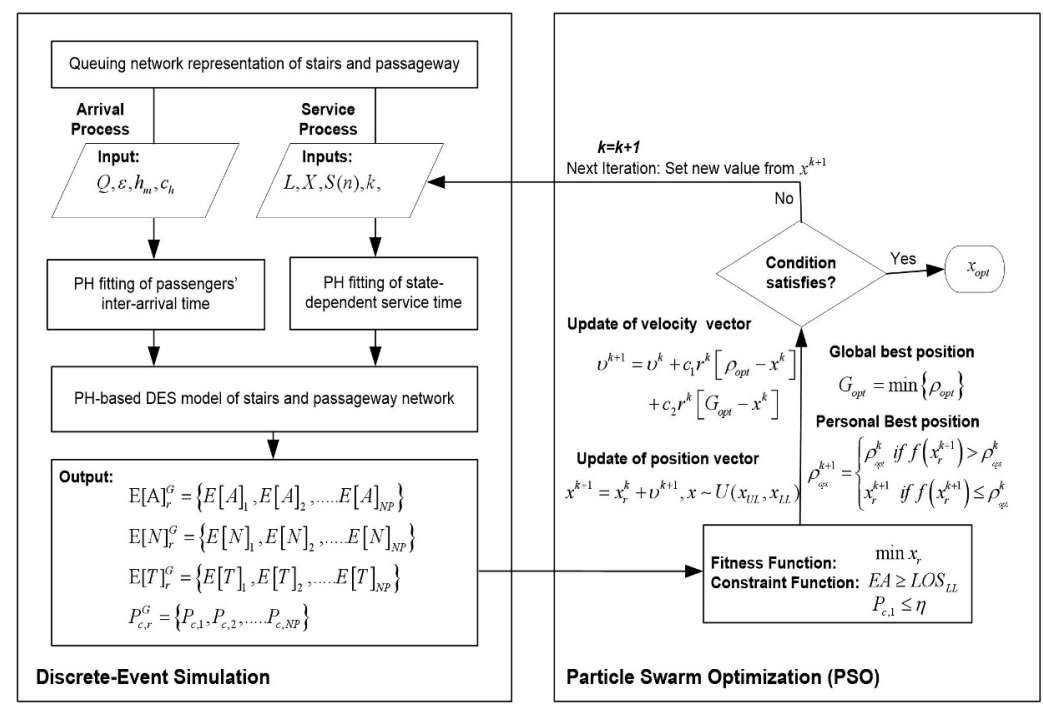

Fig. 4. Automated breadth design simulation-PSO technique.

The proposed framework is automated and iterative system that chooses the best value of breadth from the search space of particles' position $X=\left\{x_{1}, x_{2} \ldots x_{N P}\right\}$ by satisfying the range of Level of Service (LOS) (TCRP-165 Report). It also ensures that no blocking occur in the circulation area. The objective function is based on the mean area occupied per passengers $_{(E S(X))}$, obtained from the DES model.

$$
\min Z=\left(E S(X)-\operatorname{LOS}_{L R}\right)
$$




$$
\begin{array}{ll} 
& E S(X) \geq \operatorname{LOS}_{L R} \\
\text { s.t; } & E S(X) \leq \operatorname{LOS}_{U R} \\
& P_{c}(X) \leq \phi
\end{array}
$$

In PSO technique, the population is initialized by random parameter values and update in consecutive iterations until a termination condition satisfies.

\section{Computational Experiments}

The results are obtained and compared with the deterministic TCRP Report-165 which is analogous to $\mathrm{D} / \mathrm{D} / \mathrm{N} / \mathrm{N}$ model and exponential-M/G(n)/N/N model. The representative data for breadth design is obtained during morning peak (7:30-8:30am) and evening peak (17:30-18:30pm) from Chengdu Tianfu square station and Beijing Chongwanmen station, as both are the interchange stations. The lengths of both stairs and passageway are kept constant equal to $10 \mathrm{~m}$ for all experiments. The breadth design in this research is carried out under the Level of Service (LOS) B, C and D described by TCRP Report-165.

The experimental results reveal that optimal breadths values are larger for high arrival rates. It is clear from figure 5 that the Stair 2 on Line 2 has a high arrival rate that causes higher value of breadth than Stair 1 on Line 1. The optimal breadth of passageway is more than the breadths of two stairs. The reason is merging of arrival rates towards a single passageway in the direction towards line 3. A passageway with a larger breadth reduces the congestion issue and avoids blocking of passengers

With increase in SCV, the breadth increases while it remain constant for exponential$\mathrm{M} / \mathrm{G}(n) / \mathrm{N} / \mathrm{N}$ model and TCRP Report-165 technique. It is due to the fact that our proposed simulation-PSO technique accounts for the fluctuation in arrival rate and the dwell time circulation area. In existing model, the randomness is ignored and the optimal breadth value remains same. The breadth for both stairs and passageway decreases from LOS B towards $\mathrm{D}$ for all the design models. In future, several other service facilities such as; network of ticket facilities and platform can be evaluated in similar manner. In addition to that, the bidirectional flow of passengers will be incorporated in future studies.
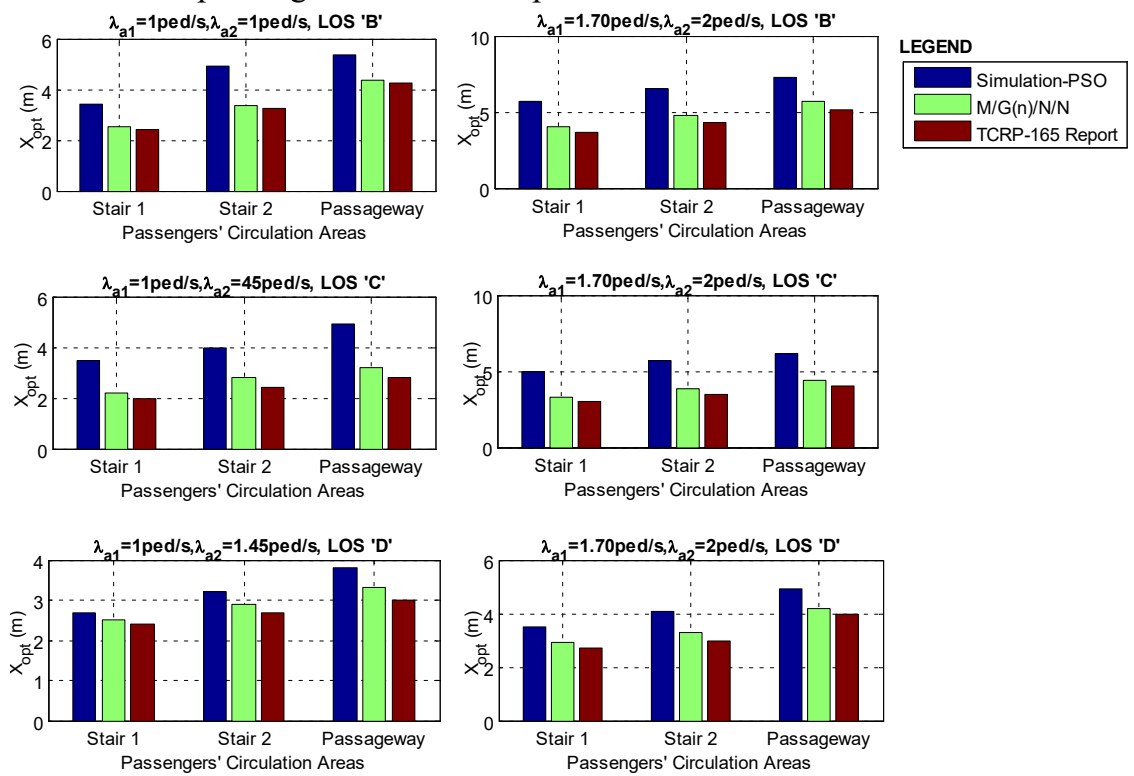

Fig. 5. Optimal breadths comparison by different design techniques. 


\section{Concluding Remark}

The breadths of network of stairs and passageways at transit station are designed by a simulation-PSO technique. The proposed model outclassed the TCRP Report-165 and $\mathrm{M} / \mathrm{G}(n) / \mathrm{N} / \mathrm{N}$ model. Our proposed simulation-based model is a hybrid and automated breadth design framework and more realistic, which considers several influential factors that were neglected by existing models. The increase in SCV value depicts the actual condition in interchange station that causes increases in the breadth of stairs and passageway under different LOS.

\section{Acknowledgment}

We express the earnest acknowledgment to the colleagues of National United Engineering Laboratory of Integrated and Intelligent Transportation at School of Transportation and Logistics, Southwest Jiaotong University, Chengdu for valuable guidance and support.

\section{References}

1. Brinckerhoff, P., Group, K., \& Texas, A. Transit capacity and quality of service manual Transit Cooperative Highway Research Program (TCRP) Report 165:(2013) Transportation Research Board Washington, DC.

2. Hu, L., et al., A PH/PH state-dependent queuing model for metro station corridor breadth design. European Journal of Operational Research, 2015. 240(1): p. 109-126.

3. Jiang, Y., L. Hu, and L. G, Determined method of subway footway breadth based on queuing theory. Journal of Traffic and Transportation Engineering, 2010. 10: p. 61-67.

4. Cruz, F.R.B., J. MacGregor Smith, and R.O. Medeiros, An M/G/C/C state-dependent network simulation model. Computers \& Operations Research, 2005. 32(4): p. 919-941.

5. Khalid, R., et al., A Discrete Event Simulation Model for Evaluating the Performances of an M/G/C/C State Dependent Queuing System. PLoS ONE, 2013. 8(4): p. e58402.

6. $\mathrm{Xu}, \mathrm{X} .-\mathrm{y}$. , et al., Analysis of subway station capacity with the use of queueing theory. Transportation Research Part C: Emerging Technologies, 2014. 38: p. 28-43.

7. Artalejo, Jesus R., and Antonio Gomez-Corral. "Modelling communication systems with phase type service and retrial times." IEEE Communications Letters 11.12 (2007).

8. Fackrell, Mark. "Modelling healthcare systems with phase-type distributions." Health care management science 12.1 (2009): 11.

9. Neuts, M.F., Matrix-geometric Solutions in Stochastic Models: An Algorithmic Approach. 1981: Dover Publications.

10. Kennedy, James. "Particle swarm optimization." Encyclopedia of machine learning. Springer US, 2011. 760-766. 